\title{
BELLEZA Y ELOCUENCIA, O LA RENDICIÓN POR LOS SENTIDOS (Lucianus, Scyth. 11)
}

\author{
MontSerRat Jufresa - FranCESCA MeStre - PILAR GóMEZ
}

Universidad de Barcelona

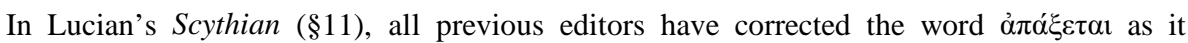
appears in the manuscripts to $\dot{\varepsilon} \pi \dot{\alpha} \xi \varepsilon \tau \alpha$. We aim to justify the restoration of the original text because, obviously, it is possible in terms of form, and, besides, it seems more appropriate to the context. The verb ,págomai implies the notion of being moved from one place to another, while $\dot{\pi} \pi \alpha \dot{\gamma o \mu \alpha}$ expresses only the notion of simple attraction which does not involve actual movement. This notion of true surrendering through the senses seems to match the idea developed here by Lucian; loci paralleli in Lucian himself and other Attic authors help us to confirm this interpretation.

El párrafo 11 de El escita o el cónsul de Luciano ${ }^{1}$ ha suscitado particularmente nuestro interés cuando hemos estudiado el texto de este opúsculo para preparar su edición ${ }^{2}$. Parece cierto que todos los manuscritos ofrecen la lección $\dot{\alpha} \pi \alpha \dot{\xi} \xi \tau \alpha$ tal como se comprueba por la lectura de aquellos a los que hemos podido acceder directamente, a saber $\mathrm{G} \mathrm{y} \mathrm{N}^{3}$, y como sugiere la falta de

1 Es la obra $n^{\mathbf{o}} 68$ de la edición de M.D. MacLeod, Luciani Opera, Vol. III, Oxford, 1980, siguiendo el orden del manuscrito G (Vaticanus Graecus 90).

2 Luciano. Obras, vol. III, texto, traducción, introducción y notas a cargo de M. Jufresa, F. Mestre y P. Gómez, Madrid, CSIC, 2000.

3 Dado que la colación exhaustiva de todos los manuscritos no nos era posible, elegimos G (s. X) porque, a partir de Nilén (cf. Lucianus, 2 vols., Leipzig, 1906-1923) es EMERITA. Revista de Lingüística y Filología Clásica (EM) — LXVIII 2, $2000 \quad$ pp. 269-278 
indicaciones contrarias en las ediciones más importantes de la obra de Luciano. Sin embargo, a partir de una primera enmienda de Valckenaer, seguido por Dobree ${ }^{4}$, los editores, casi sin excepción, dan por sentado que la lección $\alpha \dot{\pi} \alpha \dot{\xi} \xi \varepsilon \alpha \alpha$ es imposible y la corrigen en $\varepsilon \dot{\pi} \alpha \dot{\xi} \varepsilon \tau \alpha \iota^{5}$.

El contexto en que aparece dicha forma verbal es el siguiente. Luciano según él mismo refiere en el $\S 9$ - se halla en una ciudad de Macedonia, tal vez Tesalónica o quizás Béroe, $\mathrm{y}$, con objeto de ilustrar mediante un ejemplo su propia situación, dedica los capítulos iniciales de la obra a relatar qué le ocurrió al escita Anacarsis cuando llegó a Atenas. Y fue que aquél en aquella ocasión encontró en la ciudad ática a un compatriota suyo, Toxaris, a quien tomó como guía para conocer todo lo que había venido dispuesto a aprender, deseoso de instruirse en el modo de vivir de los griegos, como muy bien ilustran, asimismo, otras dos obras de Luciano, tituladas, precisamente, Toxaris, una, y Anacarsis, la otra. Toxaris no renuncia a tamaño honor, pero estima más oportuno que sea Solón el que instruya y eduque al recién llegado Anacarsis, ya que según afirma «en él se encuentra Grecia entera y podrías ya conocer lo mejor de lo bueno que hay en ella» (§ 5). Explica Luciano que Solón no defraudó la confianza de Toxaris y que, en consecuencia, Anacarsis no sólo lo aprendió todo de un único hombre, sino que fue objeto de la admiración y confianza de todos por su relación con el sabio legislador.

De la comparación con lo ocurrido a los escitas en Atenas, Luciano deduce cuán más afortunado que el propio Anacarsis ha sido él mismo en

considerado el más completo testimonio de la familia g ya que contiene 83 obras y es el mejor y más antiguo para 59 de ellas; por otra parte, elegimos N (Parisinus Graecus 2957) porque, a pesar de ser uno de los más recientes (s. XV) y considerado mixto y aun deterior por muchos autores, guarda significativas conexiones con la parte perdida $\left(\mathrm{E}^{1}\right)$ del más antiguo manuscrito de Luciano, el manuscrito E (Harleianus 5694).

4 L.C. Valckenaer, Opuscula philologica, critica, oratoria, Leipzig, 1808. Cf. también P. P. Dobree, Adversaria, Cambridge, 1831, p. 563.

5 Cf. C. Jacobitz, Lucianus, 4 vols., Leipzig, 1836-1841 [editio maior] donde sigue todavía la lección de los manuscritos mientras que en Luciani Samosatensis Opera, 3 vols., Leipzig, 1851-1857 [editio minor] incorpora la lección resultante de la enmienda de Valckenaer; G. Dindorf, Luciani Opera, col. Firmin-Didot, París, 1884²; K. Kilburn, Lucian, vol. VI., Londres-Cambridge (Mass.), 1959; E. Steindl, Luciani Scytharum Colloquia quae scribuntur Toxaris, Scytha, Anacharsis cum scholiis, Leipzig, 1970; M.D. MacLeod, cit. 
Macedonia, ya que al buscar «quiénes eran los preeminentes, y a quiénes uno podría acercarse y tener por protectores» (\$10), no ha encontrado una persona así, sino dos y de una misma familia, padre e hijo. Del padre sólo dice que hay que imaginarlo como un Solón, un Pericles o un Arístides, referentes, claro está, que hablan por sí solos.

En cambio, es precisamente en la evocación, más detallada, del hijo donde se plantea el problema textual. Luciano quiere destacar el poder cautivador de este joven y lo refiere tanto a su aspecto físico como a su elocuencia:

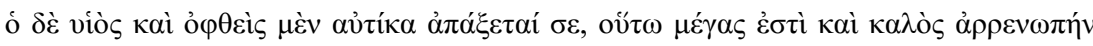

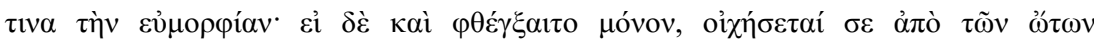

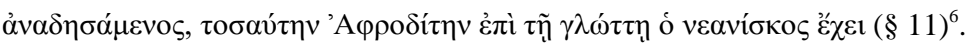

La descripción del joven abarca un período sintáctico organizado en dos secuencias unidas por la correlación $\mu \varepsilon \grave{\varepsilon} v / \delta \grave{\varepsilon}$; el hijo (ó viòs) es el sujeto de

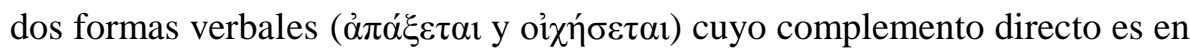
ambos casos el pronombre $\sigma \varepsilon$. A su vez, uno y otro predicado verbal están determinados por una oración circunstancial, aunque se produce una variación sintáctica. En el primer caso, dicho valor circunstancial viene dado por un participio apositivo al sujeto (ỏ $\varphi \theta \varepsilon \grave{s}$ ), pero el uso de un participio pasivo produce, por así decirlo, un quiasmo sintáctico, ya que el sujeto, activo, de la oración principal (ó viòs) pasa a ser sujeto paciente del participio, mientras que el complemento agente, no explícito, del participio,

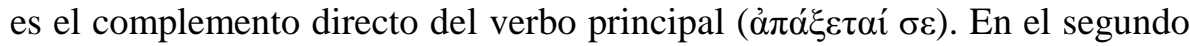
predicado, el valor circunstancial se expresa mediante una oración

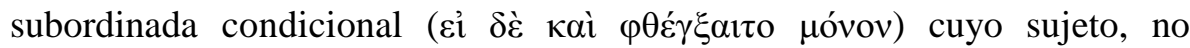
explícito, sigue siendo ó viòs.

Por otra parte, Luciano hace del joven una descripción muy sensual, en la que implica varios sentidos corporales - la vista y el oído, claramente, y quizás el gusto - y, sin duda, tiene en cuenta el papel activo que el hijo, en tanto que sujeto - gramatical también - ejerce sobre el objeto representado por el pronombre $\sigma \varepsilon$, como se comprueba en la segunda parte de la descrip-

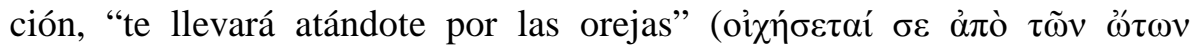
$\dot{\alpha} v \alpha \delta \eta \sigma \alpha \dot{\mu} \mu \varepsilon v \sigma \varsigma)$.

\footnotetext{
6 “... y el hijo, en cuanto lo veas te arrastrará: tan alto y tan hermoso es en sus proporciones varoniles. Y si además también se pone a hablar, te llevará atándote por las orejas: tanta es la Afrodita que tiene en la lengua ese joven".
} 
Basándonos, precisamente, en el paralelismo entre ambas proposiciones principales creemos que hay que mantener la forma utilizada por Luciano se-

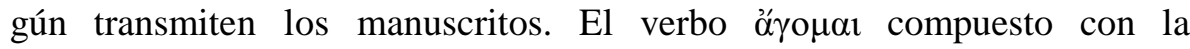
preposición غ̇ं ì - tal como corrigen los editores - indica la atracción, de algo o de alguien que, hacia sí y en su propio interés, ejerce el sujeto, valor reforzado en este caso por el uso del verbo en voz media. Este es el significado que hallamos en otros autores áticos. Sirvan como ejemplo, un pasaje de Tucídides y otro de Jenofonte. Cuenta aquél que los megarenses se sublevaron, «tras conseguir ayuda de los corintios, sicionios y epidaurios»

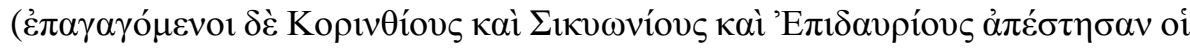

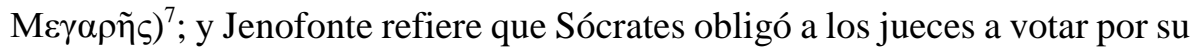
condena, «con el odio que se atrajo por aquello de ensalzarse a sí mismo en el

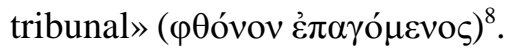

No obstante, en el pasaje de El escita el contexto tal vez permita desplazar esta perspectiva, ya que, si bien el verbo principal del primer período lleva un régimen en acusativo - el complemento directo $\sigma \varepsilon-$, éste es el agente implí-

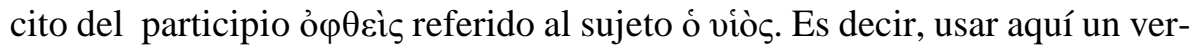
bo compuesto con la preposición ảđó permite a Luciano situar, semánticamente, la acción desde el objeto directo, que es quien resulta desplazado de su lugar - y se mantiene así el valor originario del preverbio: "con movimiento desde"".

Así pues, no se trata tanto de significar la atracción hacia, como el desplazamiento desde, expresión que encaja en el contexto, puesto que, en esta primera parte de la descripción, tal como decíamos, el joven macedonio, sujeto, no actúa propiamente, sino que se limita a "ser visto"10.

7 Th. 1.114; cf. también 1.81, 5.45.

$8 \quad \mathrm{X} .$, Ap. 32.

9 Cf. F. Rodríguez Adrados, Nueva sintaxis del griego antiguo, Madrid, 1992, p. 726, donde se expone claramente que en los verbos compuestos con preposición no siempre el primer elemento determina al segundo, sino que el preverbio puede subrayar la realización completa de la acción verbal, convirtiéndose entonces en un procedimiento para marcar un matiz aspectual. Es decir, cuando el compuesto presenta un complemento, no es determinante que éste sea precisado por el preverbio (cf. también I. Rodríguez Alfageme, Nueva gramática griega, Madrid, 1988, pp. 57-59).

10 La visión es, para la belleza física, un tópico: la belleza de alguien da placer al contemplarla y éste, sin duda, es el valor de ỏ $\varphi$ cį̀ en este pasaje, del mismo modo que el 
Por otra parte, cada una de las secuencias sintácticas de la descripción termina con una oración asindética que, a modo de explicación, justifica la

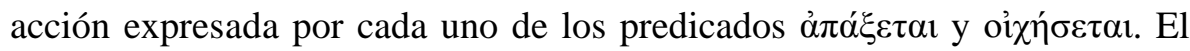
sujeto de ambas oraciones es el macedonio, elíptico en la primera y representado en la segunda por el sintagma ó veavíokos. También entre estas oraciones hay una variación sintáctica: la primera de ellas se inicia con el ad-

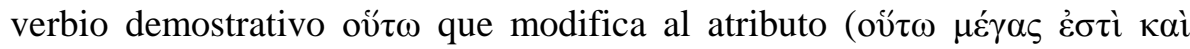

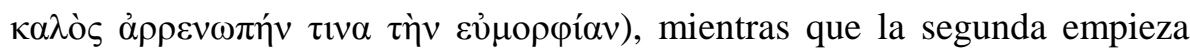

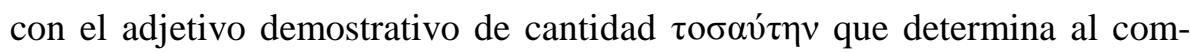

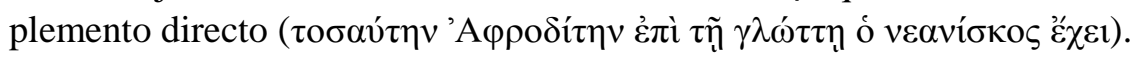

En cuanto a su contenido, la primera oración explicativa detalla la capacidad de atracción del joven relacionada con el sentido de la vista: su apariencia exterior, su belleza física son tales que mueven, desplazan, a quien lo ve, tal como se ha expresado en la frase anterior de la misma secuencia ${ }^{11}$. No es de extrañar, pues, el uso por parte de Luciano de la forma verbal $\alpha \dot{\alpha} \alpha \dot{\xi} \varepsilon \tau \alpha$, que resaltaría la intensidad del desplazamiento, máxime si se tiene en cuenta que se trata de un verbo cuyo significado genérico de "llevar consigo" o "llevarse consigo" se puede concretar, cuando es usado en contextos de contenido amoroso o erótico, en el sentido de "tomar esposa", e incluso "seducir" o "raptar" ${ }^{12}$. Además, no hay que olvidar que el referente histórico con el que es comparado el joven macedonio es Alcibíades, como comentaremos más adelante.

Las obras del propio Luciano ofrecen diversos ejemplos cuya comparación con el pasaje de El escita permite, a nuestro entender, mantener la lectura $\alpha \dot{\pi} \alpha \dot{\xi} \xi \varepsilon \tau \alpha$ de los manuscritos.

substantivo $\theta \alpha \mu \alpha ́$ en Platón (Prt. 218 d-e), del que se hablará más adelante. En este sentido, sorprende, por inapropiada en el contexto, al reflejar escasamente el juego entre la voz pasiva del participio y la voz media del verbo principal, así como el valor claramente estático de la contemplación de la belleza, la traducción de J. Zaragoza, Luciano. Obras III, Madrid, 1988, p. 490: «el hijo en cuanto te vea se hará amigo tuyo: tan alto, es, tan guapo con belleza varonil, que con sólo hablar te dejará atado por las orejas, por el atractivo que tiene el jovencito en la lengua».

11 Como reproduce la traducción de K. Kilburn, cit., p. 255: «The son will win your heart as soon as you see him, so tall is he and handsome with his manly grace».

12 Cf. s.u. ả $\pi \dot{\gamma} \gamma \omega D G E$, vol. II, Madrid, 1986, pp. 371-372; significado que también puede tener la forma sin preverbio, cf. s.u. ó $\gamma \omega$, ibidem, vol. I, Madrid, 1980, pp. 41-42, mientras que este sentido de raptar no lo tendría $\dot{\pi} \pi \dot{\gamma} \gamma \omega$. 


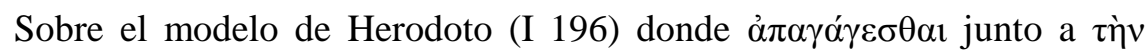
$\pi \alpha \rho \theta \varepsilon ́ v o v$ describe el acto de "tomar esposa", en la idea de que "se saca" a la doncella de la custodia o casa paterna, Luciano escribe, por dos veces en

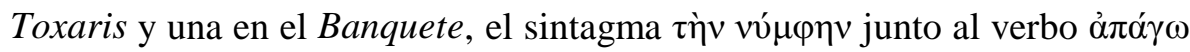
para designar la acción de "llevarse como esposa" o "dar la hija en matrimo-

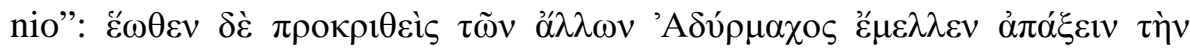
vv́ $\mu \varphi \eta$ ("por la mañana, Adírmaco, que había sido elegido frente a los

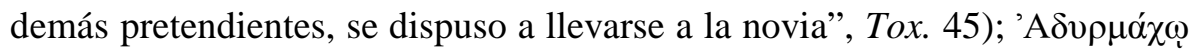

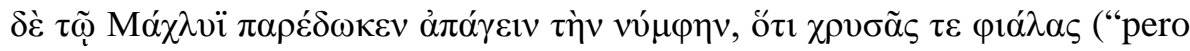

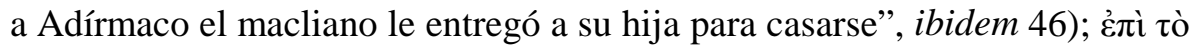

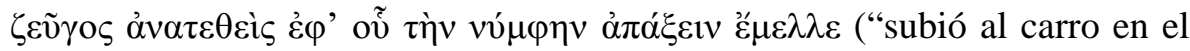
que iba a llevarse a la novia", Symp. 47).

Es más, Luciano utiliza este mismo verbo para narrar el punto final del rapto de Europa por parte de Zeus, el comportamiento indigno de Paris en el asunto de Helena o la inducción a adulterio en que puede incurrir un esposo

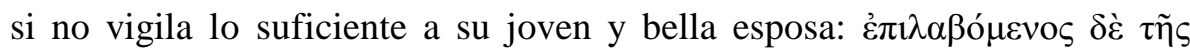

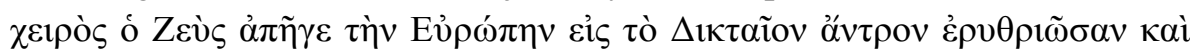

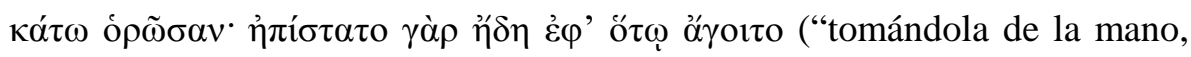
Zeus condujo a la cueva de Dicte a Europa, ruborizada y cabizbaja, pues ya

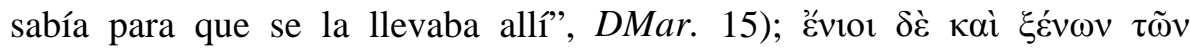

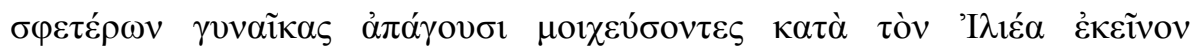

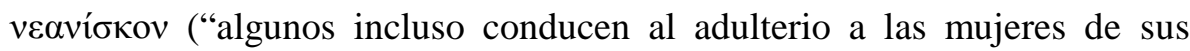

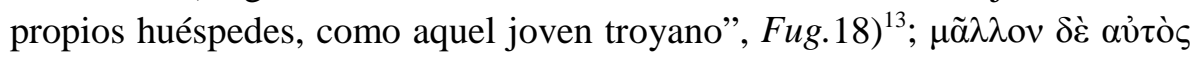

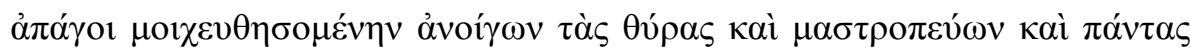

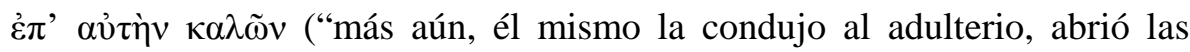
puertas y la prostituyó invitando a todo el mundo a estar con ella", Tim. 16).

La misma idea de seducción, pero con fines más perversos todavía, delata la actitud de las mujeres de la isla Cabalusa en la Historia Verdadera, donde, evidentemente, se invierten los papeles habituales de hombre/mujer, dentro del juego de múltiples inversiones que la obra presenta: $\lambda \alpha \chi 0 \tilde{\sigma} \sigma \mathrm{l} \delta$ '

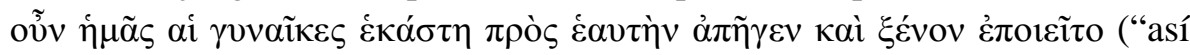
pues, cada una de las mujeres se llevó consigo, por sorteo, a uno de nosotros y lo hizo su huésped", $V H$ 2.46).

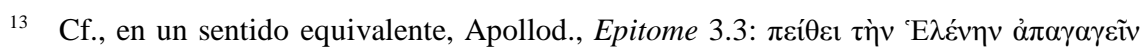
бòv $\dot{\varepsilon} \alpha v \tau \tilde{\omega}$; cf. también Parth. IV, X y XIII. 
Y también mediante este verbo ảંó $\gamma \omega$ pueden ser referidos la seducción, el rapto o el ultraje de muchachos con fines, parece deducirse, pedofílicos ${ }^{14}$ :

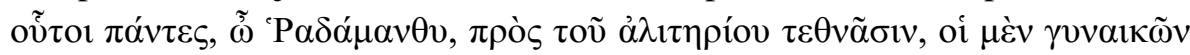

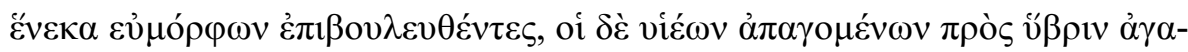
$v \alpha \kappa \tau \eta ́ \sigma \alpha v \tau \varepsilon \varsigma$ ("todos estos, Radamantis, han perecido por obra del malvado, unos víctimas de conspiraciones a causa de sus hermosas mujeres, otros de

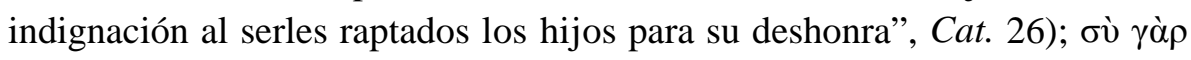

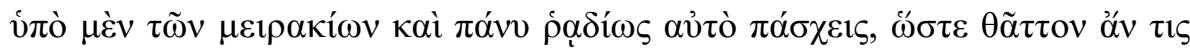

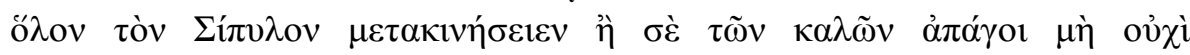

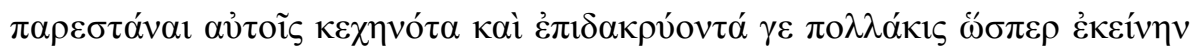

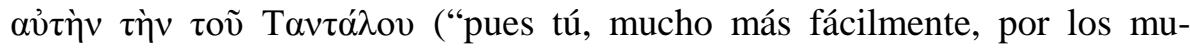
chachos experimentas esto mismo, hasta el punto de que con más rapidez alguien movería el Sípilo entero que te alejaría de los bellos mozos sin que gritaras por no permanecer junto a ellos y lloraras abundantemente como aquella hija de Tántalo", Im. 1).

La segunda secuencia introducida por $\varepsilon \grave{i} \delta \grave{\varepsilon}$, trata de la fuerza de atracción del joven macedonio, ligada ahora a su capacidad oratoria, que Luciano considera cautivadora en extremo, ya que este joven, con solo abrir la boca, arrastra por su elocuencia "atándote por las orejas" (§ 11). De este modo, Luciano recurre a una imagen que en otra ocasión refiere de la elocuencia misma, identificándola entonces con Heracles, al amparo de una tradición distinta a la helénica, cuando describe una pintura que recuerda haber visto en la Galia. En este cuadro el héroe griego también arrastra a

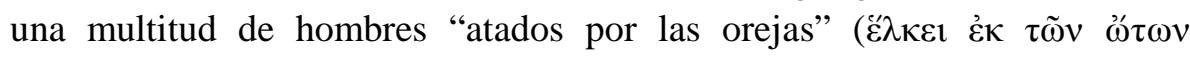

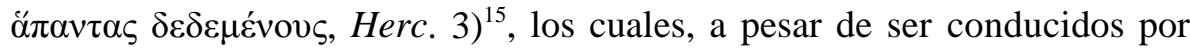
débiles cadenas, no oponen resistencia alguna. En el Proemio: Heracles, Luciano ilustra el poder cautivador de la palabra presentando como dóciles prisioneros, de verdad, a quienes escuchan al héroe, puesto que éste ha sido

14 Sin embargo, Hans Licht (seudónimo de Paul Brandt), Sexual Life in Ancient Greece, Nueva York, 1974 ( $1^{\text {a }}$ edición, Londres, 1932), p. 417, parece no estar de acuerdo con esta idea, sino que parte de la base de que, aunque se dieran este tipo de relaciones con muchachos de corta edad, éstos siempre habían iniciado la pubertad y, por tanto, no eran niños.

15 También en $J T r$. 45, Luciano reitera de nuevo esta misma imagen para designar la

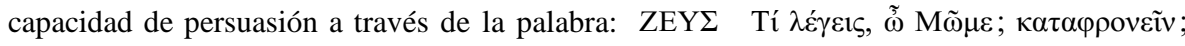

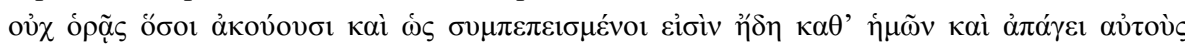

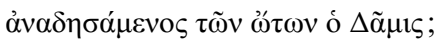


representado con las manos ocupadas por algunos de sus atributos más significativos tales como la piel del león nemeo, el arco o la maza, y, en consecuencia, los extremos de las cadenas que ligan a los cautivos de su verbo aparecen atadas a su lengua sin que ello deba producir asombro -

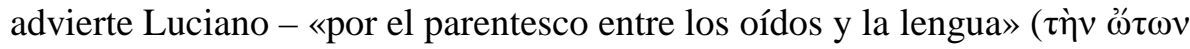

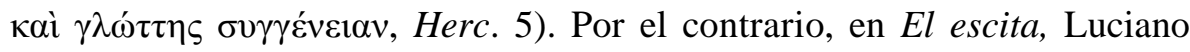
deja claro que las ataduras son sólo una metáfora para designar la fuerza seductora de las palabras del joven, cuando explica que aquéllas denotan la

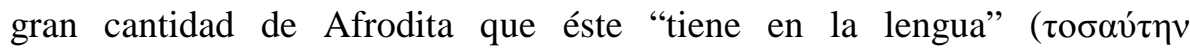

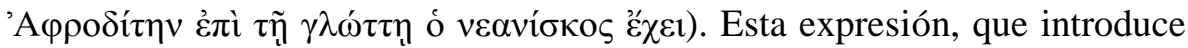
una fuerte e innegable sugerencia erótica, contribuye a justificar, creemos, el uso de $\dot{\alpha} \pi \alpha ́ \gamma \omega$ en la primera secuencia, puesto que el oyente - visualizador del hermoso y elocuente joven recibe un impacto de placer tan intenso que "consiente" en ser raptado, pues le es imposible sustraerse a la atracción que el joven le ha suscitado.

Además, si la escueta descripción del padre se basaba en una comparación con tres hombres de estado (Solón, Pericles, Arístides), emblemáticos en la historia de Atenas, tampoco falta en el retrato del hijo este referente histórico. El modelo es ahora Alcibíades cuya belleza, capacidad de seducción y habilidad oratorias son, sin duda, un lugar común en la literatura griega, de Platón y Jenofonte hasta Plutarco, pasando por los poetas cómicos y los oradores ${ }^{16}$. La elección del hijo de Clinias garantiza doblemente a Luciano el éxito en el elogio del joven macedonio, ya que, al haber sido Alcibíades tan amado como odiado por sus conciudadanos, la mayor alabanza no es ser comparado con él, sino ser considerado mejor todavía. El macedonio, como en otro tiempo Alcibíades, deja boquiabierto

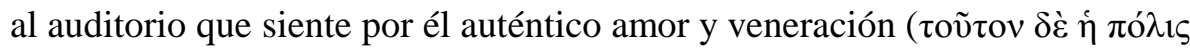

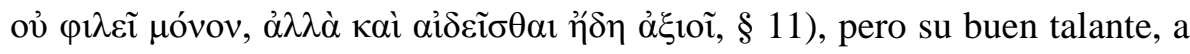
diferencia de lo que ocurrió otrora con el ateniense, hace que este amor y afecto sean perdurables.

Es interesante referirse por un instante al posible modelo platónico de la comparación con Alcibíades. En el primer intercambio de palabras al inicio del Protágoras, el interlocutor de Sócrates no tiene ninguna duda de que

16 Cf. X., Mem. 1.2.24; D. 21.145; Pl., Smp. 219; Plu., Alc. 10.4, donde se alude incluso al defecto de pronunciación que padecía. 
éste ha estado en compañía del bello hijo de Clinias, al cual, parece ser, ya le apunta la barba y empieza a tener aspecto de hombre. Esto, sin embargo, no es obstáculo para que Sócrates continúe alabando su belleza ${ }^{17}$. Ahora bien, ante la presencia de Protágoras, la visión $(\theta \alpha \mu \alpha ́)$ de Alcibíades ya no provoca nada: efectivamente, Protágoras es, para Sócrates, más bello que

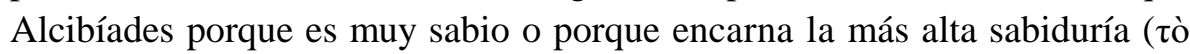

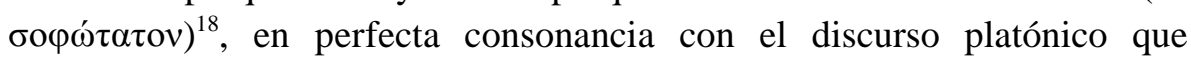
contrapone la belleza física a otro tipo de belleza. También en el Banquete (218 d-e), Sócrates, dirigiéndose precisamente a Alcibíades, establece

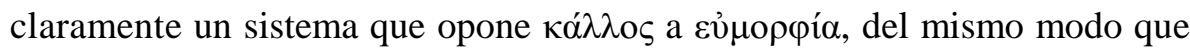
$\dot{\alpha} \lambda \hat{\eta} \theta \varepsilon 1 \alpha$ se opone a $\delta$ ó́g $\alpha$, siendo los términos de la oposición tan dispares, cualitativamente, como el oro del bronce. Y, a su vez, nos preguntamos, si, en el pasaje de Luciano objeto de nuestro comentario, la confluencia de belleza, ๕̌p $\rho \varsigma$ y sabiduría, no evocaría - de forma irónica, tan irónica que incluso el elogio queda minimizado - el contexto del mito del Fedro, donde $\kappa \alpha ́ \lambda \lambda$ os es, precisamente, la belleza cuya visión conduce a los amantes a un régimen de vida ordenado y a la filosofía misma ${ }^{19}$.

Salvando, naturalmente, todas las distancias - de género, de intención, de época -, la presentación del joven macedonio por parte de Luciano pretende englobar ambos tipos de belleza, siendo la maestría en el manejo de la palabra el equivalente, aquí, de la sabiduría.

En definitiva, pues, la lectura $\alpha \dot{\pi} \alpha \dot{\xi} \varepsilon \tau \alpha$ p parece ajustarse con más

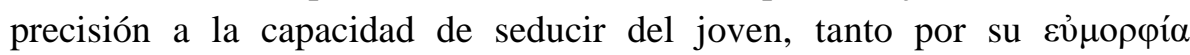
(aspecto físico) como por su $\kappa o ́ \lambda \lambda o \varsigma$ (maestría en el manejo de la palabra), cualidades que el autor describe en términos de habilidad erótica. En todo caso, y en nuestra opinión, no hay duda de que Luciano, al utilizar este verbo, lo hace de manera coherente, de acuerdo con un significado claro que corresponde a desplazamiento con finalidad de secuestro físico y espiritual. Hay que tener en cuenta, además, que Luciano tiene una gran preocupación

17 En el mismo sentido que en Il. XXIV 348 y que Estratón ( $A P$ 12.4), donde queda claro que el mejor momento para los chicos es precisamente aquél en el que empieza a despuntar la barba.

18 Cf. Pl., Prt. 309 b.

19 Cf. Pl., Phdr. 255 c. 
por el uso preciso y correcto de la lengua ${ }^{20}$. Su origen no griego, a la par que su situación militante de griego de adopción y de cultura, hacen que para él, más que para otros, éste sea un asunto de suma importancia. Es posible que cometiera o a menudo estuviera a punto de cometer errores de uso de la lengua, pero se obstina, hasta lo puntilloso, en corregirse - y corregir también a los demás.

$\mathrm{Si}$, en efecto, en este pasaje de El escita, Luciano se sirve del verbo ỏ $\pi \alpha$ -

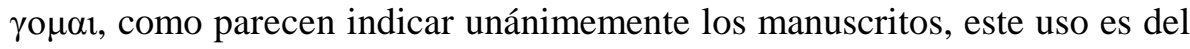
todo adecuado con el valor alusivo de la descripción del joven y la intención semántica deseada, como demuestra la presencia de este mismo verbo en otros lugares de su obra - así como en la de otros autores áticos o aticistas.

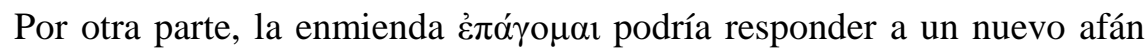
hiperaticista de los filólogos del XVIII y del XIX, seguidos por todos los demás, combinado, evidentemente, como es típico de la época, con un cierto puritanismo.

20 Cf., como obra paradigmática de esta puntillosidad, la titulada Sobre una falta cometida al saludar así como también El solecista. 